NV-25, P-18ARL, and PR70. The PR70 solar film inspired the heat rejection design in this study as it utilizes a sophisticated photoniccrystal design with alternating thin films of high- and low-refractiveindex materials forming a DBR.

The advanced engineering of materials and optimization of the optics result in $6.5 \%$ efficient solar cells and AVT of $25 \%$ with IR radiation rejection rate over $80 \%$. According to estimated calculations by Hin-Lap Yip from South China University of Technology, in a $15 \mathrm{~m}^{2}$ window area in a $100 \mathrm{~m}^{2}$ house, the ST-OPV can cut down $30 \%$ of electricity cost per household. When the ST-OPV is combined with the heat rejection properties, then the electricity reduction could reach $\sim 50 \%$. "Heat insulation is already a huge market, and [the introduction] of light harvesting will be a great added advantage," Yip says.

Kui Zhao from Shaanxi Normal University, who was not involved in this study, says, "This study has demon-

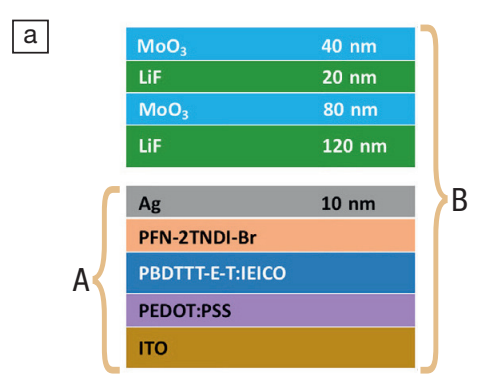

(a) Schematic of the semitransparent, organic photovoltaic (ST-OPV) device (A) and ST-OPV with Bragg reflector device (B). (b) The campus of the South China University of Technology viewed through different solar PV window films. The design of the film balances three key parameters: (1) power-conversion efficiency (PCE), (2) average visible transmittance (AVT), and (3) infrared rejection rate (HR). ITO is indium tin oxide. Credit: Prof. Hin-Lap Yip.
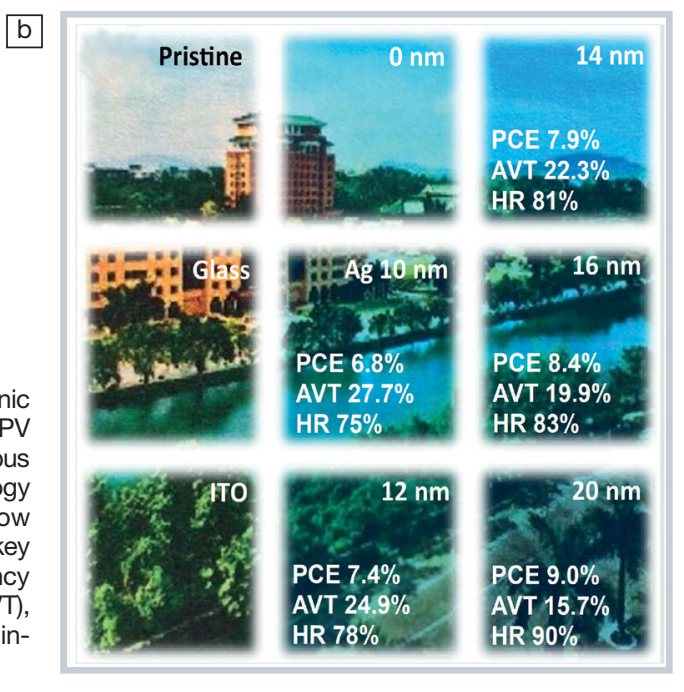

strated for the first time a dual-functional ST-OPV device. The device exhibits high performance with heat rejection properties comparable with those of commercial window films. This novel design concept is expected to open up a new approach to developing multifunctional yet efficient semitransparent organic solar cells."

Enrique Gomez from The Pennsylvania State University, who was also not involved in this study, says, "This is a clever approach to manage the optical properties of polymer solar cells that further demonstrate their potential as semitransparent windows. Although a techno-economic analysis is still needed, I expect that this approach will bring semitransparent organic solar cells closer to commercialization as window materials."

Rahim Munir

\section{Nano Focus \\ Gold nanoheaters enable optically controlled soft robots}

$\mathrm{T}$ he multicomponent structure of shapememory polymers allows different phases in the material to melt or flow at different temperatures. Subsequently, actuation of these devices requires controlled heating. Remote control using light to drive complex processes will enable new applications in soft robotics. Joseph B. Tracy from North Carolina State University, along with his former graduate student, Sumeet R. Mishra, embedded gold nanoparticles into a shape-memory polymer and achieved sequentially controlled actuation. Essential to their success was the control over geometrical shapes of plasmonic gold nanoparticles. In turn, materials dissimilarly absorb different colors of light and, subsequently, photothermally heat in fine-tuned responses to specific optical wavelengths. The researchers embedded these optically powered nanoheaters into a polyurethane shape-memory polymer, which is thermoplastic and has a component with a low glass-transition temperature, and bent strips of these materials into accordion-shaped legs. Different wavelengths of light then selectively drove the unfolding of these legs. The researchers published their discovery in a recent issue of ACS Applied Nano Materials (doi:10.1021/ acsanm.8b00394).

"This is an important advance because it directly connects the tunable optical properties of noble metal nanoparticles with remote triggering of sequential processes for applications in soft robotics, such as biomedical implants," Tracy says. "Selection

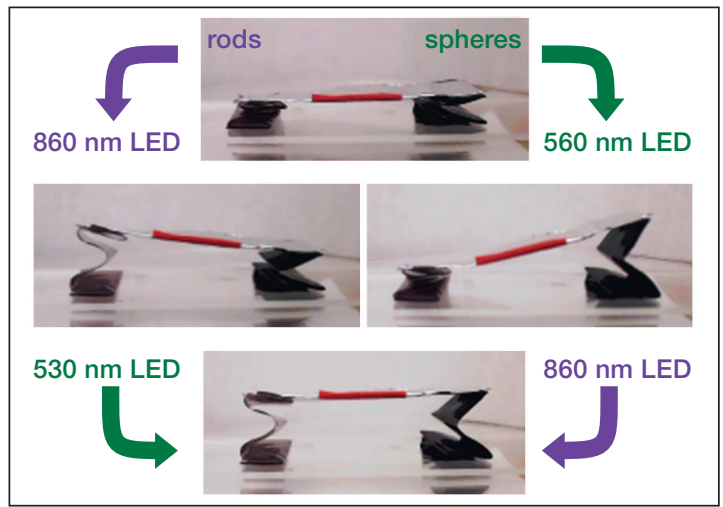

A wavelength-controlled stage with accordion legs containing gold nanorods (left) and gold nanospheres (right). The sequence of illumination by light-emitting diodes (LEDs) positioned next to each leg (not shown in photographs) remotely controls the tilt angle and height of the stage. Credit: Sumeet R. Mishra. 
Because of the small size of the gold nanoparticles $(<100 \mathrm{~nm})$ and capabilities to functionalize surfaces, they can be potentially incorporated into a wide range of polymers. The shape of the nanoparticles and their concentration adjust their sensitivity to light for driving actuation. For example, gold nanorods generate heat when illuminated by light with a wavelength of $860 \mathrm{~nm}$ (near-infrared), while gold nanospheres respond to light with a wavelength of $530 \mathrm{~nm}$ (green). A common light-emitting diode produces sufficient intensity for absorbing gold nanoparticles to control the shapes of these actuators. This advantage simplifies control over shape-memory polymer devices and makes their operation safer than lasers and less expensive.
The research team designed a stage with accordion-shaped legs and adjusted the loading of nanoparticles and illumination time to control the height and tilt angle of the top of the stage. This design approach could be adapted for more complex soft robots for a wide range of remotely controlled applications in materials and biomedical engineering.

Boris Dyatkin

\section{Nano Focus}

Remote-controlled wearable tribo-sensor is compatible with water

$\mathrm{W}$ earable electronics represents a family of smart devices that can be worn as accessories or implants, but are yet to be widely seen in day-to-day usage partly due to their incompatibility with water. Now Congju Li and his colleagues from the Chinese Academy of Sciences have designed wearable electronics that address the washability challenge.

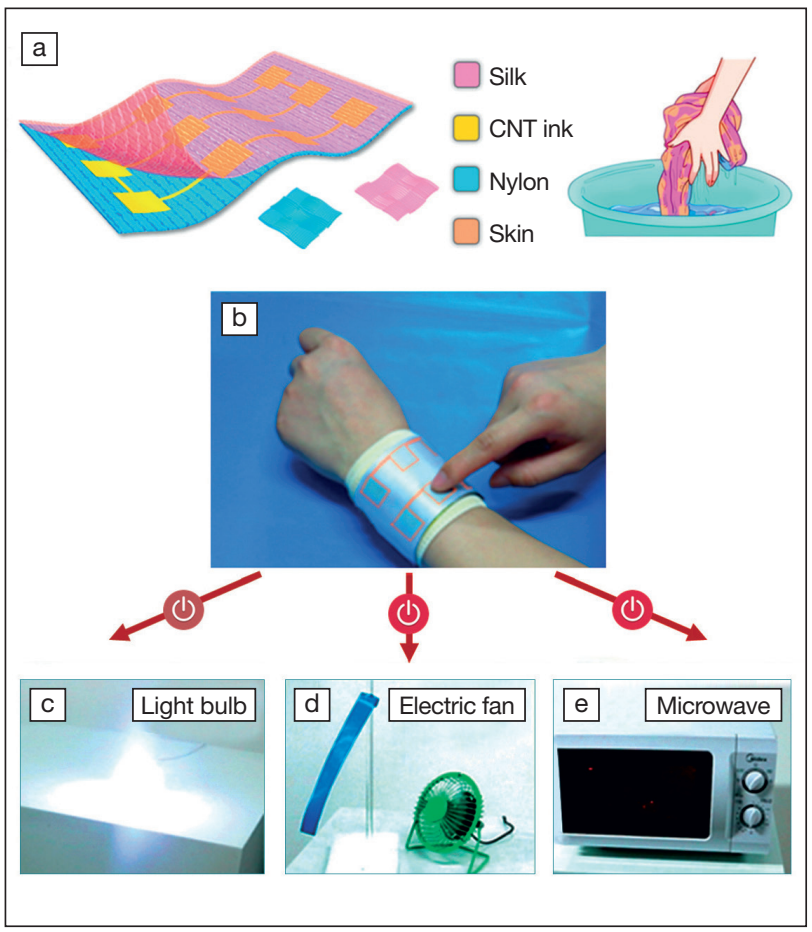

(a) Schematic showing the structure of the developed tribo-sensor. (b) Wearable tribo-sensor prototype switches on and off (c) a light bulb, (d) an electric fan, and (e) a microwave oven. CNT is carbon nanotube. Credit: American Chemical Society.
Their launderable tribo-sensor can be self-powered through the triboelectric effect and can remotely switch several home appliances on and off. This work was recently published in ACS Nano (doi:10.1021/acsnano.8b02477).

The tribo-sensor consists of three functional layers. The bottom Nylon layer serves as a flexible substrate that can be woven into clothes. The middle layer, consisting of carbon nanotube (CNT) patterns screen-printed onto the Nylon layer, functions as electric circuits. The top silk layer is a friction layer that generates and maintains electrostatic charge. To improve the adhesion between the CNT circuits and the substrate, the researchers added polyurethane to the CNT ink. Polyurethane, with its abundant amino groups, can firmly bind CNTs onto the Nylon fabric through hydrogen bonds, preventing the CNT electric circuits from detaching by washing.

The self-powering mechanism of the tribo-sensor is associated with the triboelectric effect. After frictional contact between skin and the sensor, the $\mathrm{CNT}$ and silk layers accumulate equal amounts of positive and negative electrostatic charge, respectively. Afterward, any action (e.g., touch or rub) that changes the gap between the middle and the top layers will generate electricity to power the sensor.

According to $\mathrm{Li}$, the motivation for this work is to "integrate humanmachine interfaces with clothes, which would allow convenient remote control of electronics." The researchers demonstrated how the tribo-sensor wirelessly powered three typical home appliances: a light bulb, an electric fan, and a microwave oven. The research team first incorporated the sensor as a remote controller into a wireless home control system. Touching the sensor results in a voltage output that is transmitted as electromagnetic pulse signals. These signals can then be received by the terminal electronics to turn the appliance on or off. "Imagine on a cold winter morning, you could easily turn on your TV and microwave your breakfast without leaving your warm bed," Li says.

Jun Chen of Stanford University, who was not involved in this work, says, "The screen-printed washable tribo-sensor consisting of electronic textiles for human-machine interfacing is a nice demonstration of how triboelectric nanogenerators (TENGs) can be involved in the Internet of Things. Essentially, with advantageous features including air permeability, costeffectiveness, and washability, this electronic textile-based tribo-sensor may open new ways to apply TENGs to multifunctional wearable electronic devices."

Tianyu Liu 